\title{
Le financement de la recherche dans les pays non- hégémoniques : coopération internationale et compétence nationale
}

Montserrat Alom Bartrolí, Centre International de Recherche et d'Aide à la Décision, Fédération Internationale des Universités Catholiques (FIUC)

Rigas Arvanitis, Centre Population et Développement (Ceped), Université de Paris et Institut de Recherche pour le Développement \& Global Research Institute of Paris (GRIP)

\section{DOI : 10.51186/journals/ed.2020.10-2.e353}

\section{Résumé}

La recherche scientifique est aujourd'hui une activité mondialisée qui s'effectue en réseau, autour d'objets ou de questions largement financés par des organismes internationaux, nationaux, aussi bien publics que privés. Si les partenariats des coopérations scientifiques se sont démultipliés, le mode projet, lui, s'est imposé comme mode de financement de la recherche par excellence. Cette nouvelle configuration par rapport à celle de la science du $X X^{e ̀ m e}$ siècle pose des questions sur les modalités de mise en œuvre de la coopération scientifique internationale, qui, de par sa complexité, ne peut pas se résumer à une question d'accès à des ressources financières ni de connexion à des réseaux scientifiques internationaux. Ainsi, en nous appuyant sur une enquête de terrain, la théorie de l'Acteurréseau nous permet de montrer que la participation à des projets de recherche pour le développement collaboratifs, soutenus par des financements internationaux, passe nécessairement par la construction et/ou la consolidation d'une compétence nationale. Nous discutons, en même temps, dans quelle mesure les agences qui soutiennent la recherche pour le développement contribuent à renforcer les investissements réalisés par les pays nonhégémoniques sur le plan local tout en consolidant leur positionnement à l'international.

Mots-clés : coopération internationale, recherche pour le développement, financement de la recherche, pays non-hégémoniques, théorie de l'Acteur-réseau

\section{Abstract}

Scientific research has become a globalised activity carried out in networks and around issues and questions that are largely financed by public and private international and national organizations. As partnerships for scientific cooperation have multiplied, the project mode has become the prevailing mode of research funding. This new configuration (as compared to research configurations in the 20th century) raises questions about how international scientific cooperation is carried out. Such cooperation is highly complex and cannot be reduced to a 
question of access to financial resources or connection to international scientific networks. This article, which is based on field observation, uses actor-network theory to show that taking part in internationally funded, collaborative development research projects necessarily involves building and strengthening a country's national competence. It also discusses the extent to which agencies that support development research help increase the investments of nonhegemonic countries at the local level while at the same time strengthening these countries' international position.

Keywords: international cooperation, research for development, research funding, nonhegemonic countries, actor-network theory

\section{INTRODUCTION : UN CONTEXTE INTERNATIONAL PROFONDÉMENT MODIFIÉ}

L'exercice de la recherche a subi d'importantes transformations ces dernières décennies, à l'aune du changement de paradigme qui s'est produit dans l'ensemble du régime de production des connaissances au Nord (Pestre, 2003) comme au Sud (Arvanitis \& O'Brien, 2019). Nous sommes passés, à la fin du « court vingtième siècle », d'un régime caractérisé par un mode d'institutionnalisation de la recherche s'inscrivant dans un cadre national et public, par la création et la consolidation de communautés scientifiques nationales organisées sur une base disciplinaire (Gaillard, et al., 1997), à un régime mondial où apparaît une multiplicité d'acteurs et actrices nouvelles à la fois comme exécutant-es de la recherche et comme bailleurs de fonds. Ainsi, à côté des agences de financement publiques et des organismes internationaux, des fondations privées, des organisations non gouvernementales (ONG), des entreprises ont fait leur entrée, définissant une nouvelle économie de la recherche (Vessuri, 2017).

Le début du XXlème siècle a vu l'avènement d'un monde multipolaire, où des pays comme la Chine, le Brésil, I'Inde, l'Afrique du Sud et le Mexique, parmi d'autres, se sont démarqués sur la scène internationale de par leurs moyens, leur production et leur influence sur le plan scientifique, affichant des politiques qui placent le développement de la recherche parmi leurs priorités nationales (Hanafi \& Arvanitis, 2016). Ainsi, entre 1996 et 2013, le Brésil, l'Argentine et le Mexique ont connu presque un doublement de leur population scientifique (de 115 millions équivalent temps-plein à 234 millions) et l'impact de leurs publications (selon la base de données Web of Science) a connu une forte croissance (UNESCO, 2016, p.188). La Chine devient dès 2013 le pays avec le plus grand nombre de chercheurs et de chercheuses (1,48 millions en équivalent plein temps -ETP-) très en avant par rapport à l'Inde (0,19 millions ETP). Les anciens pôles hégémoniques comme les USA (1,26 millions chercheurs en ETP), I'Europe (Union Européenne, plus la Suisse, la Norvège et l'Islande ; 1,79 millions ETP) et le Japon (0,66 millions ETP) perdent leur importance numérique relative même s'ils demeurent des puissances scientifiques par l'importance de leurs institutions, leurs entreprises de niveau mondial et la part occupée dans les publications. 
C'est pour rendre compte de ce nouveau contexte géopolitique (Barré, 2020) et contourner les représentations liées à la pauvreté qui sont associées à l'expression "pays en développement " que le concept de " pays non-hégémoniques " a été proposé (Losego \& Arvanitis, 2008). II s'applique à des pays qui n'appartiennent ni aux vieux centres ni aux nouvelles économies émergentes et qui n'ont pas d'instruments financiers capables d'influencer les grandes tendances de la production du savoir à l'échelle mondiale, mais qui possèdent toutefois des marges de manœuvre, d'une part à l'échelle nationale, pour élaborer leur propre politique scientifique et, d'autre part, dans les choix des sujets et des partenaires dans les collaborations internationales. Ce concept permet de questionner le modèle du centre-périphérie, fondé sur une dichotomie qui a dominé les études sur la coopération scientifique avec le Sud (Katz \& Bell, 1997).

La coopération scientifique internationale, elle aussi, déjà fortement accrue pendant les années 1990 (Gaillard, 2010) est devenue un mode d'exercice de la recherche pratiqué aujourd'hui par l'ensemble des pays de la planète, bien qu'à des degrés variables (Gazni, et al., 2020 ; Leydesdorff \& Wagner, 2008). Certains pays non-hégémoniques, notamment de petite taille, parient lourdement sur ce mode de fonctionnement pour assurer leur survie, devenant pratiquement dépendants du soutien des fonds de financement étrangers; c'est le cas de nombreux pays en Afrique comme le Niger (Grégoire \& Marou Sama, 2017), la Tanzanie (Koch \& Weingart, 2016), l'Ouganda (Wagner, 2008) ou même le Kenya (Lutomiah, 2019), alors que ceux à revenu moyen peuvent souvent participer à des programmes de coopération bilatéraux ou multilatéraux avec des organismes intergouvernementaux - Banque Mondiale, Union Européenne, Association des Nations de l'Asie du Sud-Est (Arvanitis \& O'Brien, 2019). De plus, l'apparition de nombreuses agences nationales de la recherche dans le Sud et la consolidation des fonds existants a changé la donne : les pays arabes témoignent d'un désir de souveraineté et d'échange, de construction de capacités domestiques et d'orientation nationale des efforts de recherche (Currie-Alder, et al., 2018) et, en Afrique sub-saharienne, plus de 50 fonds nationaux publics ont vu le jour en l'espace de quelques années seulement (Mouton, et al., 2015). Même si les montants sont parfois modestes, ces fonds publics affirment la volonté de l'État de soutenir une nécessaire capacité de recherche locale. En même temps, les fonds étrangers de financement de la recherche font progressivement l'objet d'une gestion partagée, associant à la fois des structures nationales et internationales.

Les modalités de coopération pratiquées aujourd'hui sont aussi diverses que les bailleurs présents sur la scène internationale et cela modifie le sens de la recherche pour le développement $(\mathrm{RpD})$, qui fait l'objet de cet article. L'histoire de la $\mathrm{RpD}$ reste à écrire' mais nous pouvons distinguer au moins trois périodes. Tout d'abord, des politiques d'assistance technique où des expert-es du Nord se déplaçaient dans le Sud, puis à partir des années

\footnotetext{
${ }^{1}$ Voir Gaillard (1999) ou le volume 7 (Coopérations scientifiques internationales) de la collection : Les sciences hors d'Occident au 20ème siècle, Paris : Ed. de l'ORSTOM (en ligne: https://horizon.documentation. ird. fr/) édité par R. Waast (1996)
} 
quatre-vingt des politiques essentiellement construites autour de la notion du capacity building et de la formation des communautés scientifiques nationales et, enfin, une période nouvelle où la coopération scientifique se situe dans des contextes globaux caractérisés par la montée en puissance d'agences locales dans les pays émergents (par ex. Brésil, Afrique du Sud, Turquie) dans le cadre de politiques de science diplomacy². De plus, un agenda multilatéral et global de la recherche, qui peut coexister avec des agendas propres à chaque institution, s'est progressivement imposé d'abord avec les programmes de Global Health (pour combattre l'épidémie de $\mathrm{VIH}$ au niveau mondial), puis avec les objectifs de développement durable (ODD) destinés à tous les pays et non plus seulement aux pays du Sud.

La diversification et prolifération des acteurs et des actrices, et des sources de financement a aussi imposé le mode de fonctionnement par projet comme mode de financement principal de la recherche (Hubert \& Louvel, 2012) permettant aux agences publiques de satisfaire aux exigences de transparence dans la gestion publique et la reddition des comptes (Pollitt, et al., 2007). Cependant, ce mode de financement privilégie une succession de programmes à court terme, un régime "présentiste » de la recherche ${ }^{3}$, aux dépens d'une "stratégie scientifique soutenue et prolongée " (Hubert \& Louvel, 2012, p. 21). De plus, il met en place une mise en concurrence des chercheurs et des chercheuses et des institutions, une bureaucratisation des tâches scientifiques et une recomposition des divisions du travail donnant naissance à de nouvelles hiérarchies professionnelles. Notons que dans les pays non-hégémoniques, les effets de ces conditions d'exercice de la recherche sur les collaborations internationales à moyen et à long terme demeurent largement méconnus.

Cette nouvelle configuration de la recherche pose des questions sur les modalités de mise en œuvre de la RpD car elle modifie la géographie de la recherche par les modes d'accès au financement proposés par des agences publiques et privées qui agissent au niveau international (Boekholt, et al. , 2009), et à des réseaux largement internationalisés (Alom Bartrolí, 2018 ; Arvanitis \& Mouton, 2018 ; Arvanitis \& O’Brien, 2019 ; Lutomiah, 2019 ; Marou Sama, et al. , 2019).

\section{ACCÈS AUX FONDS DE FINANCEMENT ET AUX RÉSEAUX PAR LES CHERCHEURS ET LES CHERCHEUSES DES PAYS NON-HÉGÉMONIQUES}

Les débats qui opposent présence internationale et absence sur le plan local reposent sur l'idée que les chercheurs et les chercheuses du Sud qu'on qualifierait « d'internationalisé-es » seraient uniquement ceux et celles publiant dans des revues internationales et tirant des bénéfices d'ordre académique; ils et elles s'aligneraient sur les intérêts du Nord et ne chercheraient pas à tisser des liens avec les collectifs de leur pays. Ce débat a été particulièrement fort en Amérique latine (Arellano Hernández, et al., 2012 ; Beigel, 2012) où

\footnotetext{
${ }^{2}$ La revue Science \& Diplomacy de l'Association américaine pour l'avancement de la science (AAAS) offre un panorama de cette tendance. www.sciencediplomacy.org

${ }^{3}$ Voir l'analyse des régimes d'historicité de Hartog (2012).
} 
Kreimer (2019) défendait l'idée que les chercheurs et les chercheuses du Sud étaient obligées de s'associer à des réseaux portés par le Nord sous la forme d'une "dépendance subordonnée ». Pour les pays africains, ce débat a été éludé en partie à cause de la désinstitutionalisation de la recherche jusque dans le milieu des années 2000 (Mouton, 2018) ; les pays les plus pauvres ont dû nécessairement s'appuyer sur les coopérations internationales pour engager des recherches importantes (Grégoire \& Marou Sama, 2017), mais nombre d'universitaires ont survécu en offrant leurs services d'expert-e, en brouillant ainsi les frontières entre consultance et recherche (Olivier de Sardan, 2011).

Nous défendons l'argument selon lequel la participation durable à des projets de coopération scientifique internationale passe de nos jours par la construction au préalable d'une compétence sur le plan local. Ainsi, l'accès des chercheurs et des chercheuses des pays nonhégémoniques à des financements et à des réseaux internationaux dépendra des investissements qui sont réalisés, tout d'abord dans leurs propres pays. Pour avoir accès aux fonds internationaux, véritable « nerf de la guerre » (Louvel, 2007), il faut savoir répondre à des appels à projets ayant une durée de vie limitée à l'aide de stratégies relevant d'un processus d'apprentissage qui s'effectue sur le long terme et qui implique l'acquisition d'une bonne connaissance des modes de fonctionnement, du langage et des intérêts des agences, ainsi que des modes d'approche efficaces à leur endroit. La mobilisation et le maintien des liens tissés par le passé auprès des agences s'effectuent également sur la durée, tout comme la construction d'une légitimité scientifique sur la base des succès des projets collaboratifs précédents (Tricoire, 2011). Ces stratégies n'ont de chances d'aboutir que par la construction au préalable d'une compétence spécifique qui ne peut être que le résultat d'un processus d'investissement au niveau local en termes aussi bien individuels qu'institutionnels.

Nous insistons sur cet aspect afin de dépasser le dilemme proposé par Wagner (2008), qui part du constat que les collaborations internationales se concrétisent en des réseaux internationaux fonctionnant comme un système autocentré et autogéré, auxquels les chercheurs et les chercheuses du Sud ont intérêt à se connecter (stratégie de "link » aux ressources) pour être à même de continuer à conduire leurs recherches, plutôt que d'attendre que ne se produise un important investissement d'ordre institutionnel au niveau national (stratégie de « sink » des ressources). Or, nous défendons ici l'idée que sans cet investissement local, qui s'opère au préalable dans un cadre de recherche situé localement et s'incarne dans des institutions nationales, les chercheurs et les chercheuses ne peuvent pas disposer des moyens nécessaires à la bonne conduite des projets collaboratifs et ne peuvent pas assurer un positionnement international stable et continu. En l'absence de cet investissement, ils et elles se situent dans une position d'expert-e ou de consultant-e plutôt que de partenaire scientifique, c'est-à-dire qu'ils ou elles participent du fait d'une compétence individuelle et non pas grâce à leur capacité à formuler des projets de recherche collectifs (Alom Bartrolí, 2018 ; Arvanitis, 2011). 
Dans notre analyse, nous mobilisons le concept de l'Acteur-réseau qui permet de dépasser l'opposition signalée par Wagner (2008) entre réseaux internationaux et institutions de recherche locales. En effet, la position à la fois institutionnelle locale comme scientifique (c'està-dire dans les réseaux pertinents) permet d'identifier d'autres partenaires, de mobiliser et construire des capacités de recherche et d'élargir les relations de travail et d'échange au-delà des frontières d'un territoire ou d'un laboratoire donné. La notion d'Acteur-réseau permet d'examiner ce travail à la fois local et international, donc situé à des échelles différentes (Callon \& Ferrary, 2006).

\section{CONSTRUIRE UNE COMPÉTENCE LOCALE ET INTERNATIONALE}

Notre argument repose sur plusieurs enquêtes de terrain réalisées par les auteur-es dans le but de mieux comprendre les dynamiques gouvernant le financement et les modalités de mise en place de la coopération scientifique à destination des pays non-hégémoniques, notamment des enquêtes sur les collaborations scientifiques entre chercheurs et chercheuses de l'Union Européenne et des pays hors de l'Union, en Amérique latine et dans le monde arabe, et des travaux sur les transformations des systèmes de recherche en Afrique et à travers le monde (Arvanitis, et al., 2013 ; Arvanitis \& Mouton, 2018 ; Arvanitis \& O'Brien, 2019 ; Gaillard \& Arvanitis, 2014 ; Gaillard, et al., 2013 ; Kleiche-Dray, 2017).

Nous nous concentrerons ici sur les stratégies des chercheurs et des chercheuses vis-à-vis des agences de financement de la recherche dans les coopérations scientifiques avec les pays du Sud en sciences sociales ayant fait l'objet de la recherche doctorale d'Alom Bartrolí (2018). Cette recherche repose sur les données issues d'une soixantaine d'entretiens semi-directifs auprès de chercheurs et de chercheuses du Nord (19 répondants) et du Sud (40 répondants) ayant participé à 25 projets de coopération Nord-Sud et Sud-Sud. La population d'enquête est constituée de chercheurs et de chercheuses travaillant à l'université, dans des agences gouvernementales, des ONG et des think tanks. Les projets étudiés ont été financés par trois agences présentant des politiques de recherche, des modes de fonctionnement et des rapports avec le Sud différents : la Commission européenne, le Centre de Coordination de la Recherche de la Fédération Internationale des Universités Catholiques (CCR), et le Centre de Recherches pour le Développement International (CRDI) canadien.

Notre approche, en termes de stratégies des chercheurs et des chercheuses, offre une nouvelle perspective de la $\mathrm{RpD}$, en allant au-delà de la seule opposition entre centre et périphérie et de la nécessaire dépendance des chercheurs et des chercheuses vis-à-vis de leurs partenaires du Nord. L'analyse permet de comprendre le comportement stratégique des bénéficiaires et des agences les finançant. Elle permet aussi d'examiner la forme que prennent ces réseaux d'échanges quant aux modalités de coordination, de logiques relationnelles et de dynamiques d'apprentissage comme le proposent Estades, et al. (1996). 
Pour les besoins de cet article, nous nous concentrerons sur deux agences : le CCR et le CRDI. Le CCR est une agence de la Fédération Internationale des Universités Catholiques, institution réunissant plus de deux cents universités catholiques à travers le monde (Aparicio Gómez \& Tornos Cubillo, 2014), qui finance exclusivement des coopérations Sud-Sud de RpD. Suivant une démarche top-down, le CCR adresse une invitation aux universités membres qu'elle souhaite associer à ses projets, en mentionnant parfois des chercheurs et des chercheuses. L'agence entretient ainsi un réseau de recherche, les liens se tissent et se renforcent au fil des rencontres amenant à la création d'une « clientèle » (au sens d'habitué-es à revenir vers l'agence) ; il y a là cet apprentissage de type relationnel qui permet d'affiner la connaissance mutuelle des compétences et des besoins des parties impliquées. Entretenir un réseau d'affilié-es dans une logique de proximité correspond au modèle qu'Estades, et al. (1996) appellent la forme du « club » où les partenariats se concrétisent grâce à des « relations interpersonnelles entre des acteurs qui se connaissent »(Estades, et al., 1996, p. 399).

Le CRDI est une Société d'État canadienne qui finance la RpD dans le but de produire des connaissances utiles aux politiques publiques. Dès sa création, en vertu d'une loi adoptée par le Parlement du Canada en 19704, l'agence est devenue «(...) l'une des premières organisations au monde à s'être spécialisée dans le soutien des activités de recherche que les pays en voie de développement considèrent être les plus pertinentes à leur prospérité, à leur sécurité et à leur équité » (CRDI, 2008, p. 1). L'agence finance en priorité des chercheurs et des chercheuses du Sud provenant du monde universitaire, de la société civile, du secteur public ou privé. Pendant longtemps, elle faisait figure d'exception dans le monde de la RpD car, contrairement aux grands opérateurs (coopération française, USAID, agences britanniques, etc.) qui finançaient plutôt des recherches collaboratives entre leurs nationaux et les pays du Sud, le CRDI soutenait des projets issus du terrain pour créer des capacités de recherche. Si le CRDI fonctionne à l'aide d'appels à projets concurrentiels, des logiques de proximité tiennent également une place importante dans sa stratégie : il affiche un modèle que I'on peut qualifier d'hybride entre le club, comme le CCR, et un modèle bureaucratique, comme celui de la Commission Européenne, caractérisé par une forte division du travail scientifique, la standardisation et la formalisation des procédures, la hiérarchie en termes de supervision et de centralisation des décisions (Walsh \& Lee, 2015).

Dans notre enquête, nous avons identifié des profils de bénéficiaires de financements de recherche assez typiques par les liens qu'elles et ils entretiennent avec ces agences. Dans la suite nous présentons deux de ces profils, l'un pour le CCR, l'autre pour le CRDI, tout en retraçant le processus de construction d'une compétence au niveau individuel, mais également collectif (équipe de recherche) et institutionnel, permettant de s'assurer une place au sein de la coopération scientifique internationale.

\footnotetext{
${ }^{4}$ Voir le site web du CRDI : https://www.idrc.ca/fr/propos-du-crdi
} 


\subsection{Sergio, l'archétype du chercheur d'une petite université de province engagé sur le plan local}

Notre premier profil est illustré par Sergio, un chercheur colombien d'une petite université de province. Notons que la majorité de nos répondant-es provient d'institutions académiques, qui constituent traditionnellement les lieux dévoués à la recherche. Ce sociologue de formation est enseignant-chercheur depuis environ vingt ans, bien qu'il ne soit pas titulaire d'un diplôme de doctorat, ce qui est fréquent dans les institutions d'enseignement supérieur latinoaméricaines $^{5}$. II est l'exemple du chercheur détenant un emploi stable dans une université du Sud au sein de laquelle il a pu évoluer. Comme plusieurs chercheurs et chercheuses de pays non-hégémoniques, Sergio n’a pas pu se spécialiser sur un seul sujet ; il a néanmoins su se faire un nom dans le monde scientifique colombien :

Je suis dans la base de données des évaluateurs de COLCIENCIAS [principal organisme public colombien responsable de la politique scientifique du pays] qui est l'institution qui gouverne la recherche au niveau de l'enseignement supérieur. J'évalue souvent des projets de recherche soumis par mes pairs, des résultats de recherche, des articles à publier, des communications à des colloques.

II constitue l'exemple du chercheur engagé sur le plan local que les agences finançant la RpD tendent à valoriser :

Je travaille habituellement avec des ONG, et je suis actuellement consultant pour la Mairie qui a un programme qui inclut un volet sur les travailleurs du sexe. (...) Je suis également consultant pour une institution qui a un projet visant 4 municipalités (...) sur le renforcement des compétences des entrepreneurs sur le plan familial, personnel et entrepreneurial.

Sergio a pu participer à plusieurs projets financés par la coopération internationale, notamment grâce à l'appartenance de son université à la Fédération Internationale des Universités Catholiques (FIUC). Les relations interinstitutionnelles et interpersonnelles qui se sont tissées au fil du temps avec son Centre de coordination de la recherche, le CCR, ont assuré à Sergio et à son université un soutien financier, administratif et technique récurrent :

J'ai eu la chance d'avoir travaillé sur les 5 projets qui ont été portés par le laboratoire. D’abord, celui sur le développement des jeunes, puis j'ai travaillé sur celui portant sur les migrations, puis les déplacements, un autre projet portant également sur les migrations et maintenant nous travaillons sur les questions liées à la pauvreté. J'ai donc participé à cinq projets, j'ai 15 ans d'expérience avec la FIUC.

Une première participation réussie place les chercheurs et les chercheuses dans un cercle vertueux qui se renforce au fil des collaborations. Le soutien récurrent de l'agence explique le parcours ascendant de Sergio qui est passé du statut de chercheur associé à celui de responsable de projet.

\footnotetext{
${ }^{5}$ La principale raison en est que les doctorats dans cette génération d'universitaires se faisaient le plus souvent à l'étranger. Cette situation change à partir des années 2000 (voir Gaillard \& Arvanitis, 2014).
} 
Si l'agence de financement choisit des bénéficiaires possédant des compétences solides, elle peut également contribuer à la construction d'une compétence dans un nouveau domaine. En témoigne une invitation adressée à l'équipe de Sergio pour étudier la question des migrations internationales qui ne faisait pas partie, à l'époque, de ses sujets d'expertise. Comme le reconnaît son collègue Alfonso, l'invitation a été :

(...) vécue comme un défi par l'équipe. Les membres impliqués dans ce projet ont dû se former, ont dû étudier afin de pouvoir aborder cette thématique, ont dû trouver des expertes sur le sujet (...). Cela a été un vrai défi au niveau de la formation et au niveau de l'identification car nous n'avions pas assez de connaissances sur la thématique principale.

Dans notre enquête, tous les cas indiquent que les universités membres de la Fédération n'hésitent pas à saisir les opportunités qui leur sont offertes, en mobilisant les équipes ayant déjà participé aux projets du CCR. Elles sont conscientes que la réponse continue aux propositions du CCR permet d'entretenir et de renforcer les liens. Cette logique de proximité (Estades, et al., 1996) favorise les relations interpersonnelles fondées sur la confiance. De même, avec le temps, les chercheurs et les chercheuses acquièrent de très bonnes connaissances sur le mode de fonctionnement de l'agence, son langage et ses intérêts et sont à même de répondre à ses attentes de façon satisfaisante.

En dépit donc de difficultés rencontrées au démarrage, la participation à ce premier projet sur les migrations a permis à l'équipe de Sergio de construire une compétence dans un domaine du savoir méconnu à ce moment-là :

Quant au groupe de recherche auquel j'appartiens, cela nous a permis de créer un axe de recherche, qui s'appelle 'Individus, développement et contextes d'exclusion'. En fait, nous avons appelé 'Mobilité de la population' l'axe thématique afin de couvrir non seulement la migration, mais aussi le déplacement de populations, qui est une problématique très présente en Colombie, notamment due à la violence. Avoir étudié différentes facettes de la mobilité de la population nous a permis ainsi de nous positionner par rapport à plusieurs problématiques de taille.

Sergio et son équipe ont fini par se forger une réputation et une légitimité vis-à-vis de leurs compatriotes sur les questions liées à la mobilité sur le plan international, mais également national :

Nous avions déjà conduit plusieurs projets en matière de migrations et cela nous a permis d'assurer une présence dans l'espace public local en tant qu'expert-es universitaires. En fait, lorsqu'un événement va avoir lieu en ville, nous sommes invité-es, parfois en tant qu'intervenant-es, parfois en tant qu'assistant-es.

Tous les bénéficiaires du CCR racontent un même cheminement où l'expérience s'accumule au fur et à mesure des projets et où l'agence identifie des équipes avec lesquelles elle a engagé des collaborations fructueuses. Ce cheminement est stratégique car il permet de minimiser les 
risques qu'entraîne tout projet de recherche (Tricoire, 2011). Pour le CCR, comme d'ailleurs pour toutes les agences, l'expérience préalable a une grande valeur (Quéré, 2001). L'agence perçoit ces chercheurs et chercheuses du Sud comme des personnes-ressources prisées, des points de passage obligés suivant Callon (1986), et a tendance à les solliciter fréquemment. Leur crédibilité s'accumule ainsi dans le temps (Latour \& Woolgar, 1979). Les chercheurs et les chercheuses perçoivent nettement ce processus et l'assimilent à la formation d'un pouvoir d'agir (selon la traduction de l'empowerment proposée par Camden \& Ridde (2009)) leur permettant de renforcer leurs compétences. Or, l'appartenance à la Fédération ne présuppose pas l'accès automatique aux projets coordonnés par le CCR. Seulement ceux et celles possédant les compétences requises y accèdent. C'est donc à la fois une stratégie d'intéressement de la part de l'agence qui soutient des chercheurs et des chercheuses sur les thématiques qu'elle veut favoriser (celles du monde catholique), et des chercheurs et des chercheuses qui travaillent à devenir des référent-es sur ces thèmes.

\subsection{Ignace, un chercheur aux multiples réseaux internationaux en phase avec les besoins de son pays}

Ignace, de nationalité sénégalaise, est économiste de formation. II a obtenu son Master et son doctorat aux États-Unis, après quoi il est rentré dans son pays d'origine. Notons que la moitié de nos interviewé-es ont réalisé leurs études de doctorat ou leur post-doc à l'étranger, notamment dans un pays du Nord. Comme l'affirment Gaillard, et al. (2014), « bien souvent, et assez logiquement, la mobilité professionnelle internationale des chercheurs commence bien avant le début des collaborations internationales. La première étape de cette mobilité vise l'obtention du doctorat (PhD) et/ou la réalisation d'un post-doctorat » (p. 26). La valeur ajoutée d'une formation à l'étranger a été documentée non seulement sur le plan académique, mais également humain (Tarrant, et al., 2014). Bien qu'lgnace soit parti se former à l'étranger, il s'est délibérément spécialisé en économie de l'agriculture, un domaine qu'il considère en phase avec les caractéristiques de son pays :

Je pense qu'avoir fait des études en économie agricole est un véritable atout dans mon pays parce que nous sommes un pays qui dépend de l'agriculture. Avoir donc des compétences en économie agricole fait de vous quelqu'un de précieux. (...) II n'y a pas beaucoup de gens bien formés dans ce domaine. Nous avons beaucoup d'économistes en économie de la santé ou en macroéconomie, mais très peu en économie agricole.

Ce choix stratégique lui a permis de rejoindre, dès son retour, un think tank local focalisé sur l'étude des zones rurales: parfait accord du candidat et de l'institution. Son parcours est hautement internationalisé, marqué notamment par sa participation à de nombreuses coopérations internationales :

J'ai participé à de nombreux projets de recherche internationaux. En ce moment, je participe à un grand projet sur le changement climatique avec de nombreux autres pays d'Afrique de l'Ouest et des États-Unis, d'Asie et d'Amérique latine. Je suis également impliqué dans l'après-2025... et nous avons souvent des projets avec des pays asiatiques et des pays 
d'Amérique latine. J'ai donc l'habitude de participer à des projets avec des collaborateurs d'autres pays.

Le positionnement d'Ignace à l'international est toutefois tributaire de celui de son institution. Les projets que son think tank a remportés lui ont permis de rentrer dans un cycle qui est allé crescendo. D'un projet à l'autre, ses connaissances et compétences se sont retrouvées renforcées. Rappelons que traduire c'est, entre autres, " exprimer dans son propre langage ce que les autres disent et veulent » (Callon, 1986). Désormais, Ignace est à même de traduire les intérêts de ses différents bailleurs :

Eh bien, il est difficile d'obtenir des fonds, mais si vous avez les compétences et que vous connaissez très bien les intérêts du bailleur de fonds, vous pouvez préparer de bons projets, des projets qui sont pertinents, et vous pouvez obtenir du financement. Et si vous pouvez établir de bonnes relations avec des coalitions, avec des centres de recherche, alors vous pouvez obtenir du financement sans souci. Vous devez être malin et vous devez avoir une bonne équipe. Et une fois que vous avez un projet, vous devez également assurer. Si vous êtes capable d'assurer, vous avez beaucoup de chances de remporter un autre projet par la suite.

Si la construction de réseaux est un élément clé permettant de bénéficier de fonds, la connaissance du terrain et le travail accompli sont des prérequis essentiels qui témoignent du sérieux de l'institution et de son personnel. Jouent également un rôle incontournable les stratégies des chercheurs et de chercheuses pour s'approprier et redéfinir les cadres cognitifs des projets proposés par les agences afin de faire converger les intérêts de toutes les parties impliquées (Alom Bartrolí, 2018). Par ailleurs, en accord avec la nature de son institution, la compétence qu'lgnace a su forger en termes de valorisation des connaissances produites explique en grande partie le soutien du CRDI :

En tant que think tank, notre objectif consiste à faire de la recherche, mais les résultats de nos recherches doivent nous permettre d'alimenter le dialogue politique, d'organiser des débats, d'aider le gouvernement et les autres parties prenantes à mieux comprendre les enjeux. (...) Nous avons même réalisé un projet pour l'État sur la jeunesse. Nous leur avons fourni beaucoup de données pour les aider à comprendre ce qui se passait sur le marché du travail rural. Ce n'était donc pas seulement de la recherche : c'était de la recherche, du lobbying et du dialogue.

De nos jours les agences doivent répondre aux demandes sociales et politiques d'obtention de résultats rapides et concrets, et de reddition de comptes. Le CRDI, qui est redevable au Parlement canadien du fait de l'histoire de sa création, s'intéresse à des thématiques globalisées répondant à la fois aux besoins des pays du Sud ${ }^{6}$ et aux priorités du Canada (CRDI, 2013). II finance des recherches ayant des retombées concrètes sur la société des pays du Sud, notamment sur leurs politiques publiques. II y a ainsi une convergence d'intérêts certaine

\footnotetext{
${ }^{6}$ || possède quatre bureaux régionaux (Égypte, Uruguay, Kenya, Inde) afin d'être au plus près des besoins locaux.
} 
entre le mandat d'institutions comme les think tanks et celui du CRDI pour qui l'interaction avec les pouvoirs publics s'avère fondamentale.

De plus, la présence de think tanks est récurrente parmi les bénéficiaires du CRDI en raison de leur nombre et importance grandissante dans le monde, en Afrique sub-saharienne y compris (le Kenya en compte 53, le Nigéria 48, le Ghana 37, l'Ouganda 28). Selon le classement international Think Tank Index, les think tanks de 5 pays africains figuraient parmi les meilleurs au monde en 2016 et, au Sénégal, une quinzaine de think tanks se sont constitués en réseau en 2019 afin d'influer davantage sur l'agenda politique national. Le CRDI a lui-même promu I'Initiative Think Tank (2009-2019) visant à assurer la pérennité de 43 think tanks en Amérique latine, Asie du Sud et Afrique subsaharienne, dont celui de notre répondant ${ }^{7}$.

La compétence scientifique qu'Ignace a pu construire au fil des années et les réseaux tissés avec le monde politique sénégalais sont indissociables des conditions fournies par le contexte institutionnel dans lequel il exerce son activité. Ce sont des résultats de sa participation continue à des projets s'insérant dans le cadre de la coopération internationale.

\section{CONCLUSION : COMPRENDRE LA COOPÉRATION AU-DELÀ DE L'OPPOSITION ENTRE DÉPENDANCE INTERNATIONALE ET CONSTRUCTION LOCALE}

Les débats sur la recherche scientifique dans les pays non-hégémoniques reposent notamment sur une vision opposant nécessairement compétence locale et positionnement international, dans un monde divisé en pays du centre et pays périphériques. C'est particulièrement vrai de la circulation des publications comme l'a résumé Hanafi (2011) dans la formule "Publish globally and perish locally vs Publish locally and perish globally ». Mais, en observant les modalités de participation à des projets de coopération scientifique financés par des fonds internationaux, tous les témoignages que nous avons pu recueillir soulignent la nécessité d'un investissement local important comme préalable à une présence durable dans des réseaux internationaux. Dans les deux cas examinés dans ces pages, nous avons deux modalités différentes de cet investissement local : dans l'université pour Sergio et dans un think tank à visée politique pour lgnace. Des récits signalant de la même façon la nécessité de cet engagement et permanence dans une institution de type académique ont aussi été recueillis dans d'autres ouvrages (voir interview de Abdu Salam Fall, dans Vidal, 2014 ; Droz, 2009 ; Tidjani Alou, 2009). Au travers de la participation dans les projets financés, l'apprentissage acquis dans la relation aux bailleurs de fonds permet d'obtenir une compétence particulière sur les formes de soutien, la manière de répondre aux appels à projet, le langage de chaque agence.

Pour les agences qui promeuvent la RpD, nous constatons une tendance à soutenir financièrement les mêmes institutions et équipes, soit d'un projet à un autre, soit de façon discontinue, mais récurrente dans le temps. Ce soutien régulier s'explique par une volonté de

\footnotetext{
${ }^{7}$ Site web dédié au projet : http://www.thinktankinitiative.org/fr
} 
contribuer au renforcement et à la consolidation des capacités de recherche d'institutions et d'équipes ayant fait preuve de sérieux et de professionnalisme, la construction d'une compétence scientifique s'opérant dans le temps. Ce soutien s'explique aussi par le fait que, dans le Sud, il y a souvent un nombre réduit de spécialistes dans un domaine donné. Si d'éventuelles dérives en termes de clientélisme ne doivent pas être sous-estimées, il n'en reste pas moins que les agences jouent un rôle non négligeable en ce sens qu'elles permettent de soutenir et renforcer des acteurs et actrices locales par le biais de la coopération internationale. Ainsi dans le cas d'Ignace, nous sommes ici très loin des chercheurs et des chercheuses africain-es qui peinent à trouver des subventions pour subsister ou qui se retrouvent pris-es dans des relations de dépendance vis-à-vis des financements extérieurs ${ }^{8}$; ou encore, avec Sergio, nous sommes loin de ces chercheurs et chercheuses latino-américain-es qui seraient cantonné-es à une dépendance subordonnée aux seules volontés de leurs partenaires du Nord (Kreimer, 2019) $)^{9}$.

Nous avons également constaté que nombre de répondant-es travaillent sur des thématiques d'intérêt national et entretiennent des interactions en dehors de l'académie avec différents collectifs du pays. Cela rejoint les intérêts des agences qui soutiennent la RpD, qui cherchent à s'appuyer sur des individu-es et des institutions disposant d'un ancrage local solide, ayant tissé des réseaux sur place et étant ainsi à même de contribuer aux transformations de leur société. II est intéressant à ce sujet de rappeler une enquête de Tijssen \& Kraemer-Mbula (2017) sur le sens qu'il faut donner à l'excellence de la recherche selon les acteurs et les actrices de la recherche en Afrique du Sud ; cette dernière serait « sa capacité à résoudre un problème, à améliorer la vie des personnes (en particulier celles qui sont marginalisées ou défavorisées) ou à changer les politiques » (p. 400). C'est donc bien cet engagement dans la résolution de questions nationales qui est privilégié de nos jours par les fonds internationaux dédiés à la RpD.

\section{DÉCLARATION DE CONFLIT D'INTÉRÊT}

Montserrat Alom Bartrolí est directrice du Centre International de Recherche et d'Aide à la Décision de la Fédération Internationale des Universités Catholiques (FIUC). Rigas Arvanitis, chercheur de l'IRD, déclare avoir obtenu des financements du CRDI. Les travaux mentionnés ici n'ont été financés par aucune de ces deux institutions et ont fait l'objet des travaux de doctorat en sociologie de l'Université de Paris (anciennement Paris Descartes) obtenu par M. Alom Bartrolí.

\footnotetext{
${ }^{8}$ En Afrique, seule la moitié des répondant-es dans la grande enquête sur les chercheurs et chercheuses africaines déclarent avoir eu accès à du financement pour la recherche (Beaudry, et al., 2018, pp. 72-73). Et moins de $30 \%$ des jeunes enseignant-es-chercheurs/euses des universités africaines ont effectué un voyage à l'étranger. ${ }^{9}$ Tous les chercheurs et toutes les chercheuses ne se trouvent pas dans une situation d'égalité face à l'accès au financement en Amérique latine par rapport à leurs partenaires nord-américain-es ou européen-nes, mais comme l'a montré l'enquête EULAKS les latinoaméricain-es et européen-nes ayant participé à des collaborations internationales tendent à avoir les mêmes caractéristiques sociodémographiques (Gaillard \& Arvanitis, 2014).
} 


\section{RÉFÉRENCES}

Alom Bartrolí, M. (2018). Les stratégies d'acteurs dans les collaborations scientifiques avec le Sud. Chercheurs et agences de financement dans les sciences sociales. Thèse de doctorat de sociologie, Université Paris Descartes.

Aparicio Gómez, R., \& Tornos Cubillo A. (2014). Youth Cultures in Catholic Universities, A Worldwide Study. Paris : IFCU.

Arellano Hernández, A., Arvanitis, R., \& Vinck, D. (2012). Circulation et connexité mondiale des savoirs. Éléments d'anthropologie des connaissances en Amérique latine. Revue d'anthropologie des connaissances, 6(2), 1-28.

Arvanitis, R. (2011). Que de réseaux ! A propos du livre de Caroline Wagner : The New Invisible College. Revue d'anthropologie des connaissances, 5(1), 177-184.

Arvanitis, R., Atweh, R., \& M'henni, H. (2013). Assessing international scientific cooperation in the Mediterranean region. An international challenge ahead. In C. Morini, R. Rodriguez, R. Arvanitis, \& R. Chaabouni (Eds.), Moving to the future in the Euro-Mediterranean Research and Innovation partnership (pp. 105-131). Bari \& Paris : Options Méditerranéennes (Series B - Studies and research), CIHEAM.

Arvanitis, R., \& Mouton, J. (2018). Observing and Funding African Science. Paris : Ceped. https://www.ceped.org/fr/publications-ressources/working-papers-duceped/article/observing-and-funding-african

Arvanitis, R., \& O'Brien, D. (Eds.). (2019). The Transformation of Research in the South: policies and outcomes. Paris : Édition des Archives Contemporaines, CRDI \& IRD.

Barré, R. (2020). Pour une géopolitique de la recherche. Géoéconomie, 2, 13-51.

Beaudry, C., Mouton, J., \& Prozesky H. (2018). Lack of funding. In C. Beaudry, J. Mouton et H. Prozesky (Eds.), The Next Generation of Scientists in Africa (pp. 71-88). Cape Town: African Minds.

Beigel, F. (2012). The politics of academic autonomy in Latin America. Farnham (UK) \& Burlington (USA): Ashgate.

Boekholt, P., Edler, J., Cunningham, P., \& Flanagan, K. (Eds.). (2009). Drivers of International collaboration in research. Final Report Luxembourg: European Commission, DG Research, International Cooperation (EUR 24195).

Callon, M. (1986). Eléments pour une sociologie de la traduction : la domestication des coquilles Saint-Jacques et des marins pêcheurs dans la baie de Saint Brieuc. L'Année Sociologique, 36, 169-208.

Callon, M., \& Ferrary, M. (2006). Les réseaux sociaux à l'aune de la théorie de l'acteur-réseau. Sociologies pratiques, 13(2), 37-44. http://doi:10.3917/sopr.013.0037

Camden, C., \& Ridde, V. (2009). Le pouvoir d'agir des intervenants dans le domaine de la réadaptation. Passerelles (Montréal), 1(1), 46-65.

CRDI (2008). Report presented to the Board of Governors. Special examination report. Ottawa, ON: CRDI.

CRDI (2013). De la recherche à l'action : rapport annuel 2012-2013. Ottawa,ON : CRDI. 
Currie-Alder, B., Arvanitis, R., \& Hanafi, S. (2018). Research in Arabic-speaking countries: funding competitions, international collaboration, and career incentives. Science and Public Policy, 45(1), 74-82. http://doi:10.1093/scipol/scx048

Droz, Y. (2009). Mythes et réalités du partenariat scientifique. In Y. Droz, \& A. Mayor (Eds.), Partenariats scientifiques avec l'Afrique : Réflexions de Suisse et d'ailleurs (pp. 17-34). Paris : Editions Karthala.

Estades, J., Joly, P.-B., \& Mangematin, V. (1996). Dynamique des relations industrielles dans les laboratoires d'un grand organisme public de recherche : coordination, apprentissage, réputation et confiance. Sociologie du Travail, 38(3), 391-408.

Gaillard, A.-M., Canesse A. A., Gaillard, J., \& Arvanitis, R. (2013). Euro-Mediterranean Science and Technology Collaborations: a Questionnaire Survey. In C. Morini, et alii (Eds.), Moving to the future in the Euro-Mediterranean Research and Innovation partnership - The experience of the MIRA project (pp. 79-102). Bari \& Paris: Options Méditerranéennes (Series $\mathrm{B}$ - Studies and research), CIHEAM.

Gaillard, A.-M., Gaillard, J. \& Arvanitis, R. (2014). Hacia una cooperacion mas equilibrada entre la búsqueda de excelencia y de financiamiento. In M. Kleiche Dray, \& D. Villavicencio (Eds.) Cooperación, colaboración científica y movilidad internacional en América Latina (pp. 1948). Buenos Aires: CLACSO. http://www.documentation.ird.fr/hor/fdi:010063450

Gaillard, J. (1999). La coopération scientifique et technique avec les pays du sud. Peut-on partager la science? Paris: Karthala.

Gaillard, J. (2010). Measuring Research and Development in Developing Countries: Main Characteristics and Implications for the Frascati Manual. Science, Technology \& Society, 15(1), 77-111.

Gaillard, J., \& Arvanitis, R. (2014). Science and technology collaboration between Europe and Latin America: towards a more equal partnership? In J. Gaillard, \& R. Arvanitis (Eds.), Research collaborations between Europe and Latin America. Mapping and Understanding partnership (pp. 1-23). Paris : Éditions des Archives Contemporaines.

Gaillard, J., Krishna, V. V., \& Waast, R. (1997). Scientific communities in the developing world. New Delhi \& London: Sage.

Gazni, A., Sugimoto, C. R., \& Didegah, F. (2012). Mapping world scientific collaboration: Authors, institutions, and countries. Journal of the American Society for Information Science and Technology, 63(2), 323-335.

Grégoire, E. \& Marou Sama, K. (2017). Constitution d'une communauté scientifique dans un pays moins avancé. Le cas du Niger. In M. Kleiche-Dray (Ed.), Les ancrages nationaux de la science mondiale (pp. 423-448). Paris : Édition des Archives Contemporaines/IRD.

Hanafi, S. (2011). University Systems in the Arab East: Publish Globally and Perish Locally Vs. Publish Locally and Perish Globally. Current Sociology, 59(3), 291-309.

Hanafi, S., \& Arvanitis, R. (2016). Knowledge production in the Arab World: The impossible promise. London: Routledge.

Hartog, F. (2012). Régimes d'historicité. Présentisme et expérience du temps. Paris : Le Seuil. Hubert, M., \& Louvel, S. (2012). Le financement sur projet : quelles conséquences sur le travail des chercheurs? Mouvements, 3, 13-24. 
Katz, J. S., \& Bell, M. (1997). What is research collaboration? Research Policy, 26(1), 1-18. http://doi:10.1016/S0048-7333(96)00917-1

Kleiche-Dray, M. (2017). Les ancrages nationaux de la science mondiale. Paris : Éditions des Archives Contemporaines/IRD. http://eac.ac/books/9782813002716

Koch, S., \& Weingart, P. (2016). The Delusion of Knowledge Transfer: The Impact of Foreign Aid Experts on Policy-making in South Africa and Tanzania. Cape Town: African Minds.

Kreimer, P. (2019). Science and Society in Latin America. Peripheral Modernities: Routledge.

Latour, B., \& S. Woolgar (1979). Laboratory Life. The social construction of scientific facts. Beverly Hills: Sage.

Leydesdorff, L., \& Wagner, C. (2008). International collaboration in science and the formation of a core group. Journal of Informetrics, 2(4), 317-325.

Losego, P., \& Arvanitis, R. (2008). La science dans les pays non-hégémoniques. Revue d'anthropologie des connaissances, 2(3), 334-342. http://www.cairn.info/revueanthropologie-des-connaissances-2008-3.htm

Louvel, S. (2007). Le nerf de la guerre. Relations financières entre les équipes et organisation de la coopération dans un laboratoire. Revue d'anthropologie des connaissances, 1(2), 297322.

Lutomiah, A. O. (2019). An Evaluation of the Science System in Kenya. PhD Thesis, Stellenbosch University.

Marou Sama, K., d'Aiglepierre, R., \& Botton, S. (2019). Recherches africaines et rôles de l'aide internationale : le cas des sciences sociales. Paris : AFD.

Mouton, J. (2018). African science: A diagnosis. In C. Beaudry, J. Mouton, \& H. Prozesky (Eds.), The Next Generation of Scientists in Africa (pp. 3-12). Cape Town: African Minds.

Mouton, J., Gaillard, J., \& van Lill, M. (2015). Functions of Science Granting Councils in SubSaharan Africa. In N. Cloete, P. Maassen et T. Bailey (Eds.), Knowledge Production and Contradictory Functions in African Higher Education (pp. 148-170). Cape Town: African Minds.

Olivier de Sardan, J.-P. (2011). Promouvoir la recherche face à la consultance. Autour de l'expérience du lasdel (Niger-Bénin). Cahier d'études africaines, 202-203, 511-528.

Pestre, D. (2003). Science, argent et politique. Un essai d'interprétation. Paris : INRA Sciences en questions.

Pollitt, C., Van Thiel, S., \& Homburg, V. (Eds.). (2007). New public management in Europe. Adaptation and Alternatives. Basingstoke: Palgrave Macmillan.

Quéré, L. (2001). La structure cognitive et normative de la confiance. Réseaux, 108(4), 125152.

Tarrant, M., Rubin, D., \& Stoner, L. (2014). The Added Value of Study Abroad: Fostering a Global Citizenry. Journal of Studies in International Education, 18(2), 141-161.

Tidjani Alou, M. (2009). Des relations Nord-Sud en quête de souffle : L'exemple de la recherche en sciences sociales à travers l'expérience du LASDEL. In Y. Droz \& A. Mayor (Eds.), Partenariats scientifiques avec l'Afrique : Réflexions de Suisse et d'ailleurs (pp. 129-148). Paris : Éditions Karthala 
Tijssen, R. J. W., \& Kraemer-Mbula, E. (2017). Research excellence in Africa: Policies, perceptions, and performance. Science and Public Policy, 45(3), 392-403.

Tricoire, A. (2011). La structuration d'un projet européen : du réseau scientifique au collectif de recherche. Terrains \& Travaux, 18(1), 81-101.

UNESCO. (2016). UNESCO Science report: Towards 2030. Paris: UNESCO.

Vessuri, H. (2017). From Science as ‘Development Assistance' to 'Global Philanthropy'. In D. Tyfield, R. Lave, S. Randalls, \& C. Thorpe (Eds.), The Routledge Handbook of the Political Economy of Science (pp. 405-415). London: Routledge.

Vidal, L. (Ed.) (2014). Expériences du partenariat au Sud. Le regard des sciences sociales. Marseille : Éditions de l'IRD.

Waast, R. (Ed.). (1996). Les sciences hors d'Occident au XXè siècle: Coopérations scientifiques internationales (vol. 7). Paris : ORSTOM.

Wagner, C. (2008). The New Invisible College. Science for Development. Washington D.C.: Brookings Institution Press

Walsh, J. P., \& Lee Y.-N. (2015). The bureaucratization of science. Research Policy, 44(8), 1584-1600.

Open Access Publications - Bibliothèque de l'Université de Genève Creative Commons Licence 4.0

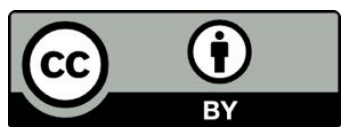

